\title{
Aux sources de la représentation des femmes autochtones dans trois récits de voyage français de la Renaissance
}

Megan Talbot, Université de Waterloo

Aborder la question de la représentation des femmes autochtones au Canada est une importante question d'actualité. Nous constatons tous les jours que les femmes des différentes premières nations sont l'objet d'amalgames et de préjugés, et sont trop souvent victimes d'ostracisme. La colonisation est bien entendu à l'origine de cette situation, et il peut paraître bizarre de toujours se pencher sur les récits de l'époque des premières explorations et des premières colonisations pour mieux comprendre ce qui, dans ce phénomène qui nous touche aujourd'hui, a pu mal tourner. Nous allons en fait analyser, dans le présent article, des descriptions de femmes autochtones élaborées dans les premières années du contact entre les Français et les Autochtones d'Amérique. Les descriptions alors élaborées sont évidemment teintées par les mentalités et les schèmes d'interprétation de l'époque, mais les auteurs ont tout de même laissé, de l'avis de plusieurs spécialistes, « des témoignages qui servent aujourd'hui de sources d'information précieuses permettant de mieux comprendre la vision de l'ailleurs à l'époque » (Kulagina 61). Nous allons ainsi examiner, dans les extraits de trois récits de voyage, comment un voyageur protestant qui séjourna au Brésil, un missionnaire récollet en Nouvelle-France et l'ursuline Marie de l'Incarnation établie à Québec semblent à la fois à se conformer à une vision stéréotypée de la femme indigène, tout en se permettant, dans le cas de Marie de l'Incarnation, des écarts intéressants d'un point de vue anthropologique. Écrits sur une période d'une centaine d'années, aux premiers temps du processus de colonisation de l'Amérique par la France, du milieu du seizième siècle au milieu du dix-septième siècle, l'Histoire d'un voyage faict en la terre du Brésil de Jean de Léry, Le grand voyage du pays des Hurons de Gabriel Sagard et les lettres de Marie de 1'Incarnation sont le reflet des premiers contacts de Français avec différentes tribus autochtones. Plus précisément, nous analyserons, dans le présent article, trois instantanés de l'image des femmes décrites par les trois auteurs. Dans un premier temps, nous comparerons comment leurs vêtements et leurs comportements sont rapportés. Ensuite, nous parlerons des devoirs des femmes dans la société autochtone. Pour terminer, nous aborderons l'héroïsme attribué aux femmes autochtones par Marie de l'Incarnation. 
À travers leur regard étranger, Léry, Sagard et Marie de L'Incarnation soulignent l'habillement des femmes autochtones qu'ils comparent à celui des Européennes. Selon Léry, les Françaises portent de «fausses perruqes, cheveux tortillez, grands collets fraisez, vertugales, robbes sur robbes, et autres infinies bagatelles dont les femmes et filles de par-deça [la France] se contrefont et n'ont jamais assez»(234). À l'époque, la culture française donne beaucoup d'importance à l'habillement, encourageant la vanité et l'affichage des richesses. Toutefois, Léry soutient que la nudité est un mode de vie dans la société autochtone, montrant un contraste dans les apparences physiques et les valeurs des deux cultures : «Au reste, chose non moins estrange que difficile à croire à ceux qui ne l'ont veu, tant hommes, femmes qu'enfans, non seulement sans cacher aucunes parties de leurs corps, mais aussi sans monstrer aucun signe d'en avoir honte ny vergongne, demeurent et vont coustumierement aussi nuds qu'ils sortent du ventre de leurs meres » $(212,214)$. Chez les femmes autochtones, les vêtements ne sont pas de grande valeur et la nudité est commune, alors qu'elle est taboue dans la société européenne. Pourtant, dans son récit, au lieu de condamner les Autochtones à cause de leur manque de pudeur, Léry observe la diversité entre les deux cultures.

Dominique Deslandres affirme que « tous les agents missionnaires vont décrire les traits, les qualités, les défauts, les forces et les faiblesses qui caractérisent les peuples qu'ils cherchent à convertir »(58). Dans son récit de voyage, le missionnaire récollet Sagard brosse le tableau des femmes autochtones en insistant sur leurs ornements du corps. Selon Sagard, les femmes portent de nombreux accessoires, y compris des colliers faits en porcelaine, des chapelets pendus à leurs oreilles, des chaînes de grains par-dessus leurs cuisses et des bracelets :

Leurs porcelaines sont diversement enfilées, les unes en colliers, larges de trois ou quatre doigts [...] puis d'autres enfilées comme nos patenôtres, attachées et pendues à leurs oreilles, et des chaînes de grains gros comme noix, de la même porcelaine qu'elles attachent sur les deux hanches [...] et j'en ai vu d'autres qui en portaient encore des bracelets aux bras et de grandes plaques par devant leur estomac. (228-229)

Bien entendu, les femmes étant nues, ces bijoux, bracelets et pendentifs deviennent leurs formes d'habillement. Sagard semble bien saisir, malgré la barrière de la langue, l'importance d'accessoires comme les ceintures. Il va d'ailleurs alors se mettre en scène, comparant sa propre ceinture $^{1}$ de disciple de saint François à celle des femmes qu'il rencontre en Amérique : « Nos sauvages ${ }^{2}$ croyaient au commencement que nous portions nos chapelets à la ceinture pour parade, comme ils font leurs porcelaines, mais sans comparaison ils faisaient fort peu d'état de nos 
chapelets, disant qu'ils n'étaient que de bois » (230). Sagard, qui porte une corde autour de ses hanches pour symboliser sa foi et son dénuement de frère prêcheur, comprend alors que les Autochtones portent des ceintures comme ornement vestimentaire. En revanche, ses observations montrent que l'inverse n'est pas vrai ; les autochtones ne comprennent pas, selon lui, la valeur symbolique du chapelet de Sagard, mais le voient tout simplement comme un objet fait de bois. Ainsi, Sagard présente non seulement son regard sur les Autochtones, mais aussi ce qu'il comprend du regard des Autochtones sur les frères récollets.

De même, Marie de l'Incarnation est moins intéressée par les parures que par la simplicité des femmes du Nouveau Monde : «quel plaisir de se voir avec une grande troupe de femmes et de filles Sauvages dont les pauvres habits qui ne sont qu'un bout de peau ou de vieille couverture, n'ont pas si bonne odeur que ceux des Dames de France ! Mais la candeur et simplicité de leur esprit est si ravissante qu'elle ne se peut dire » (108). L'auteure des lettres ne décrit pas leurs parures en détail, mais simplement les matériaux utilisés pour se couvrir et l'odeur qu'ils dégagent. Il faut garder en tête que Marie de l'Incarnation est ursuline ${ }^{3}$ et que l'abnégation domine chez elle. Comme résultat, l'apparence physique n'a pas d'importance. Marie garde ainsi une position objective et n'impose pas ses valeurs dans ses descriptions des femmes autochtones.

Si l'on fait un saut dans le temps présent, nous constatons que les vêtements et les accessoires qui leur sont liés, font encore aujourd'hui l'objet d'un écueil dans le dialogue entre Européens et Premières Nations. Thomas King explique ainsi que les représentations des Autochtones dans la littérature et dans les films, par exemple, ont créé une version stéréotypée de l'Autochtone qui se définit par les vêtements : «North America has had a long association with Native people, but despite the history that the two groups share, North America no longer sees Indians. What it sees are war bonnets, beaded shirts, fringed deerskin dresses, loincloths, headbands, feathered lances, tomahawks, moccasins, face paint, and bone chokers ${ }^{4} \gg(59)$. Il faudrait bien entendu trouver des exemples franco-canadiens, mais l'auteur souligne notamment que la culture autochtone continue à être dépeinte comme dans les anciens récits de voyage. Les films westerns, mais également des productions des studios Disney comme Pocahontas reprennent au fond, pour le grand public, des clichés créés il y a plusieurs siècles.

Pour renforcer leurs descriptions des femmes autochtones, les auteurs évoquent également leur comportement. Léry, Sagard et Marie comparent les femmes de l'Ancien et du Nouveau Mondes en faisant ressortir l'opposition entre le comportement des femmes autochtones et des 
femmes françaises. Léry et Sagard s'éloignent quelque peu de ces clichés européens en soulignant la différence entre la nudité et le désir sexuel des femmes. En particulier, Léry révèle que la nudité des femmes autochtones n'est pas attirante, mais est grossière et ne stimule pas le désir sexuel. Ce qui renforce le désir sexuel, ce sont les femmes françaises qui couvrent leurs corps avec des vêtements sophistiqués qui «cause[nt] [...] plus de maux que n'est la nudité ordinaire des femmes sauvages » (234). Ainsi, nous retrouvons un discours paradoxal puisque les femmes nues sont perçues comme moins désirables que les femmes qui couvrent leur corps. Pour les lecteurs, ce discours peut surprendre et Léry le reconnait : «je me rapporte de ce peu que j'en ay dit à ceux qui ont fait le voyage en la terre du Bresil, et qui comme moy ont veu les unes et les autres » (235). L'écriture de Léry montre qu'il est conscient que ses commentaires sur les femmes peuvent contenir des divergences et donc essaie d'éliminer toute incertitude qu'un lecteur peut y avoir.

Le discours de Sagard défie également les conceptions de l'époque en révélant que la femme autochtone «lubrique », c'est-à-dire dominée par le désir charnel, n'est qu'un mensonge. Laurier Lacroix insiste que «le missionnaire s'accommode généralement assez bien de ses conditions de vie et porte peu de jugements de valeur sur le comportement des indigènes » (332). De ce fait, Sagard est un missionnaire qui s'éloigne de ses propres valeurs et maintient une perspective dialogique. Même s’il fait alors référence, dans ce passage, à des idées développées plus tôt par Marc Lescarbot, auteur d'une Histoire de la Nouvelle-France (1609), il énumère les raisons pour lesquelles les femmes autochtones sont moins sujettes aux vices sexuels que les femmes françaises :

[...Je peux] dire, avec vérité, n’y avoir jamais vu donner un seul baiser ou faire aucun geste ou regard impudique; et pour cette raison j'ose affirmer qu'ils sont moins sujets à ce vice que par-deçà, dont on peut attribuer la cause, partie à leur nudité, et principalement de la tête, partie au défaut des épiceries, du vin, et partie à l'usage ordinaire qu'ils ont du pétun ${ }^{5}$, la fumée duquel étourdit les sens et monte au cerveau. (Sagard 203).

Puisque les femmes sont nues et leurs corps révélés, Sagard, tout comme Léry, pense que ce phénomène entraîne une luxure moins grande qu'en France. En ce qui concerne le vin et les épices, Sagard explique que cela «étourdit les sens et monte au cerveau » (203), croyance populaire à l'époque selon laquelle ces éléments en particulier peuvent stimuler la sexualité, mais comme les Autochtones n'ont pas accès à ces aliments, ils n'en ressentent pas les effets. Finalement, le pétun est populaire dans la communauté autochtone et l'on croyait que la fumée montait au cerveau, bloquant le désir sexuel. Lescarbot lui-même insiste qu'en «partie au defaut du sel, des épiceries, 
du vin, \& des viandes qui provoquent les [Autochtones], \& en partie à l'usage ordinaire qu'ils ont du Petun, la fumée duquel etourdit les sens, \& montant au cerveau empeche les fonctions de Venus » (829). À cette époque, l'usage du tabac n'est pas encore très répandu chez les Français, subséquemment il n'y a pas de fumée qui bloque leurs désirs. Sagard cherche donc, tout comme Léry, à donner un portrait très positif de la sexualité de la femme autochtone. S'il laisse entendre également que le vêtement peut susciter le désir, il suit les recommandations d'usage des confesseurs à propos des épices, stimulant, croyait-on à l'époque, la sexualité. Il est aussi important de prendre conscience que ces deux auteurs tranchent de l'image d'hypersexualité qui est associée à la femme à l'époque : «[1]a civilisation chrétienne, qui imprègne toutes les représentations mentales et sociales à la Renaissance, a imposé l'image de la femme tentatrice et corruptrice » (Lazard 17). Les femmes tentatrices qui corrompent l'homme, comme Ève qui a tenté Adam, étaient une idée reçue, à l'époque, représentée dans l'art, la littérature et la poésie. Madeleine Lazard dénonce que «[d]epuis le Moyen Age, les intellectuels et les écrivains sont pour la plupart des clercs célibataires auxquels la féminité inspire d'autant plus de mépris et de crainte » (17). Si Léry repousse cette vision misogyne sur la femme catholique (excluant la femme autochtone et, logiquement, la femme protestante), la perspective de Sagard s'avère alors fort progressiste pour un frère récollet.

Les lettres de Marie apportent également une perspective nouvelle sur le comportement des filles autochtones. Au lieu de seulement décrire les jeunes filles, dans ses lettres, de son point de vue, elle parle des jeunes filles qui posent un regard sur les ursulines. Dans la lettre 31, elle écrit à la Mère Marie-Gillette Rolland : « Il en est arrivé plusieurs d'une Nation fort éloignée, qui nous voiant étoient en peine de notre façon de vie. Ils me demandèrent pourquoi nous avions la tête enveloppée [...]. Je leur dis que les Vierges de notre païs étoient ainsi » (108). L'habillement des ursulines et des filles autochtones représente deux extrêmes. Les ursulines couvrent complètement leur corps, au contraire des filles autochtones qui portent des peaux ou sont toutes nues. Les lecteurs, qui sont Européens, voient la perspective et la curiosité des filles envers les coutumes des sœurs religieuses. De plus, contrairement à ses homologues, Marie insiste aussi sur la personnalité de plusieurs jeunes filles qu'elle décrit grâce à des anecdotes. Elle personnalise alors son regard, notamment lorsqu'elle décrit Marie Negabmat, venue au Séminaire alors qu'elle aimait courir dans les bois et n'aimait pas porter de vêtements européens. L'auteure explique dans sa lettre que Negabmat «s'enfuit quatre jours après dans les bois aiant mis en pièces une robe que nous lui 
avions donnée. Son Père qui est un excellent Chrétien et qui vit comme un saint lui commanda de revenir au Séminaire, ce qu'elle fit. Elle n'y fut pas deux jours qu'il y eut un changement admirable » (95). Dans cette description, Negabmat appartient ni plus ni moins à la nature, et en est une partie intégrante. Cependant, à partir de la lettre 41, Negabmat devient tous les jours plus religieuse : «On ne prendroit pas la petite Magdeleine pour une Sauvage, il ne se peut voir un enfant plus obeïssant, ny plus affectueux [...] c'est un petit Ange en innocence » (91). Puisque Marie est ursuline, l'emploi du terme «ange» est significatif. Negabmat devient alors la messagère de Dieu et une femme vertueuse. Claire Gourdeau dénonce que «la mission fondamentale des ursulines de Québec au dix-septième siècle [est] celle de convertir les Amérindiennes au catholicisme et de dispenser aux filles françaises une éducation de base visant à en faire des épouses vertueuses et des mères pieuses » (145). Le portrait des filles autochtones donné par Marie insiste donc sur les filles qui représentent des dévotes en formation. Elle veut montrer l'évolution des jeunes filles qui au début ont rejeté la culture européenne, comme l'habillement et les croyances européennes, mais qui l'acceptent plus tard. Même s'ils s'éloignent du tabou de la nudité, la voix masculine des descriptions de Léry et de Sagard se concentre sur l'apparence physique des femmes ; la nudité, les ornements du corps et le désir sexuel. Marie, elle, dépeint plutôt, grâce à sa voix de femme, la transformation religieuse des jeunes filles.

Pour mieux approfondir notre portrait de la femme du Nouveau Monde, il faut examiner les devoirs de la femme dans la société autochtone. Léry étend sa description sur plusieurs chapitres, présentant les devoirs mixtes des femmes autochtones. L'auteur écrit dans son texte que les devoirs de la femme sont de prendre « soin de la cuisine et du ménage » (177), « de la poterie » et des «petites provisions » (178). Comme moyen de bien expliquer les devoirs à ses lecteurs, il décrit en détail les tâches. Pour la préparation des racines, il indique que : « les femmes (car les hommes ne s'y occupent point) apres les avoir faits secher au feu sur le Boucan [...] à force de les raper sur certaines petites pierres pointues, fichées et arrengées sur une piece de bois plate [...] elles les reduisent en farine laquelle est aussi blanche que la neige » (237-238). Léry prend beaucoup de temps à expliquer la cuisine et la présente comme une curiosité chez l'auteur. Comme observateur, il est curieux de la façon dont la nourriture est préparée et des tâches des femmes. De façon semblable, Sagard évoque l'importance des femmes dans la vie quotidienne des Hurons. Elles sont à la fois mères, fermières, cuisinières et domestiques : « Elles travaillent ordinairement plus que les hommes [...]. Elles font de la poterie [...]. Elles font des nattes de joncs, dont elles 
garnissent les portes de leurs cabanes [...], elles font leurs manteaux ou couvertures [...]. Elles font semblablement des paniers de jonc, [...] elles font aussi comme une espèce de gibecière de cuir, ou sac à pétun, sur laquelle elles font des ouvrages dignes d'admiration » (177-178). Dans cette description, Sagard fournit une longue liste d'environ trois pages et répète les mots «elles font ». Sagard n'est pas simplement un religieux qui essaie de convertir les Autochtones, mais il devient aussi ethnographe. Il est un auteur qui tente de décrire la culture et les pratiques d'une société différente de la sienne. Lui-même devient observateur et essaie de transmettre cette culture à son public, au lieu de simplement écrire sur sa tentative de les convertir. De nombreux chercheurs tels que «[1]es historiens et littéraires qui se sont penchés sur ses textes remarquent que Sagard a un esprit d'observation aigu et qu'il note les détails de manière précise. Le texte est d'une grande richesse au plan ethnographique, en particulier pour l'attention que Sagard porte aux Amérindiennes » (Lacroix 328). Ainsi Sagard, comme Léry, décrit les devoirs des femmes avec de nombreux détails afin de souligner les différences entre les deux cultures. Lire le travail de Sagard en tant qu'ethnographe continue à être porteur de sens au vingt-et-unième siècle. Ces textes permettent « de préserver et de transmettre l'expérience des autres, ceux qui sont éloignés de nous dans l'espace et le temps, ou qui diffèrent de nous par les conditions de leur vie. [Ils] nous rend[ent] sensibles au fait que les autres sont très divers et que leurs valeurs s'écartent des nôtres » (Compagnon 63). Non seulement les lecteurs à l'époque, pour la première fois, vont avoir accès à des récits de voyage qui montrent l'altérité entre peuples, mais les lecteurs modernes ont l'occasion de découvrir le passé et de voir comment les gens vivaient différemment.

Marie de l'Incarnation nous révèle aussi que les femmes autochtones ont une vie spirituelle qui ressemble à celle des chrétiens. À cet égard, les filles transmettent leur foi dans leur village : « il semble que ces bonnes gens portent le Paradis avec eux, aussi sont ce des âmes fraîchement lavées dans le sang de l'agneau » (91). Ceci dit, Marie croit fermement que ces filles portent leur religion en elles, qu'elles sont pures et feront tout pour répandre leur religion. L'histoire de Marie Amiskvian est l'exemple le plus frappant. Amiskvian, qui voulait se marier à un Français, doit épouser un homme autochtone pour mieux partager sa foi. Dans son anecdote, Marie explique : « [Amiskvian] est recherchée de mariage par un François, mais on a dessein de la donner à une de sa Nation à cause de l'exemple qu'on espère qu'elle donnera aux Sauvages » (95). Les sœurs ont donc une bonne idée de l'importance du rôle religieux des jeunes Autochtones, et elles vont également les accompagner dans les premières étapes de leur vie d'adulte et de mère. L'impression 
donnée par les lettres de Marie aux lecteurs modernes est que la conversion des filles autochtones est un grand succès. Il faut prendre en considération que nous disposons seulement des histoires des Européens sur les Autochtones, ce qui rend difficile de prouver ce qui s'est vraiment passé. En tant que lecteur moderne, nous n'avons aucun moyen de savoir si les filles acceptaient vraiment la religion. Marie nous fait sûrement croire que c'est un succès, elle leur donne des noms, elle les reconnait comme individus et donne une histoire aux filles qu'elle rencontre. Elle donne un nom métissé aux jeunes néophytes, composé à la fois d'un nom chrétien, comme Marie, suivi par leur nom autochtone : Marie Negabmat, Marie Amiskvian, Marie Madeleine Abatenau et Nicole Assepanse. Elle dédie un paragraphe à chaque fille dans ses lettres. Même s'il est possible de remettre en question la véracité du contenu de cette correspondance, elle fournit des indications sur le quotidien de ses jeunes élèves.

Le thème de l'héroïsme des femmes est uniquement souligné dans les lettres de Marie. Pour plusieurs raisons, les parents amènent leurs filles au Séminaire pour les protéger. Gourdeau indique ainsi qu' « À une époque où la colonie subit la menace épisodique des Iroquois, puis, des Anglais, le souci d'assurer la protection physique des fillettes se fait pressant » (146). Dans les lettres de l'ursuline, une fille qui est trop jeune pour aller à la guerre entre les tribus ou à la pêche avec ses parents sera laissée au monastère afin que les sœurs la protègent. Gourdeau soutient aussi que les monastères sont l'«endroit sécuritaire aux plans physique et moral, le pensionnat des ursulines de Québec est aussi un lieu de refuge, un abri temporaire, une ressource d'accueil fiable pour les filles dont les familles subissent des épreuves ou vivent des moments difficiles » (148). De plus, Marie devient une figure maternelle pour les filles. Quand les jeunes filles quittent leur mère, la religieuse dit qu' « elles ne font paroistre aucun désir de les suivre, elles les saluent à la Françoise, et les quittent en riant, il semble que nous soyons leurs mères naturelles ; elles se viennent jetter en nos bras, comme à leur refuge » (91). Marie influence une grande partie de la vie des jeunes filles. Elle va apprendre le Huron, la langue maternelle des filles, pour les éduquer et les soigner comme si elles étaient ses propres enfants.

Marie va encore plus loin en démontrant l'héroïsme des femmes autochtones. Au Nouveau Monde, elle raconte qu'il y a plusieurs guerres, famines et que l'environnement dans lequel elles vivent est très dur. À ce sujet, considérons par exemple l'histoire d'une Autochtone, Marie, la femme de Jean Baptiste. Pendant la guerre entre les Iroquois, connus aujourd'hui comme les Mohawks, et leur rival, les Hurons, la femme de Jean Baptiste fuit des Iroquois qui essayent de la 
faire prisonnière. Elle se cache dans les bois avec seulement deux petits plats de blé d'Inde. Elle perd ainsi toute espérance et, sans succès, essaye de se suicider. Plus tard, la femme de Jean Baptiste trouve Dieu et un miracle se produit. Marie de l'Incarnation croit que «Dieu néanmoins qui n'abandonne jamais dans la nécessité ceux qui ont confiance en lui, permit qu'elle trouva une hache dans un lieu où les Hiroquois avoient cabané. Cet instrument lui sauva la vie » (329). Marie utilise sa hache pour se défendre et trouver de la nourriture. Après deux mois à errer dans les bois, elle arrive à son village. L'anecdote de la femme de Jean Baptiste démontre à la fois son courage face à l'adversité et le pouvoir de Dieu. En racontant les histoires des héroïnes autochtones, Marie de l'Incarnation donne une voix aux femmes en rapportant leurs histoires extraordinaires. Elle va beaucoup plus loin que Léry et Sagard dans sa défense des femmes autochtones et elle développe tout au cours de ses lettres une nouvelle perspective pour les femmes. Son but est d'aider les jeunes filles à transformer leur vie et celle de leur tribu par la religion. Le lecteur a l'impression qu'elle devient la voix des filles en représentant leur réalité et leurs histoires personnelles. Elle leur permet alors de s'imposer dans la société française à titre de modèle héroïque métissé.

Pour être plus précis, les lettres de Marie de l'Incarnation éclaircissent non seulement les événements historiques, mais le début de la colonisation. En lisant ces textes aujourd'hui, les lecteurs ont accès à un document ethnographique reflétant le quotidien de l'époque, mais aussi à des exemples de dialogues, bien timides il est vrai, entre la culture française et les cultures des Premières Nations, dialogues qui pourraient aujourd'hui nous inspirer quant aux rendez-vous ratés que l'Occident a eu, sous l'Ancien Régime et, l'histoire de notre pays malheureusement le prouvant, aux dix-neuvième, vingtième et vingt-et-unième siècles. Nous apprenons aussi, par ces textes, sur la culture religieuse et européenne des voyageurs et des missionnaires, mais également sur les schèmes de pensée qui encore aujourd'hui déterminent nos perceptions de l'altérité et, dans une certaine mesure, du racisme et de l'ostracisme.

Les trois récits de voyage du seizième et dix-septième siècles constituent un héritage littéraire qui a permis de préserver 1'histoire des premières rencontres entre les Européens et les Autochtones. Ils apportent une nouvelle perspective sur l'interaction entre des sociétés qui apprenaient à se connaître, avant le début d'une colonisation qui allait imposer les règles des nouveaux arrivants. Les œuvres de Léry, de Sagard et de Marie reflètent curiosité (pour Léry) et tentative de conversion (pour Sagard et Marie), mais intègrent des observations des femmes autochtones afin, pourrait-on croire, de mieux la circonscrire ou la comprendre. Ces auteurs 
ouvrent la porte à l'altérité de sociétés nouvelles et d'une expérience de la rencontre unique, que lecteurs de la Renaissance et lecteurs modernes découvrent. Alors que les descriptions des vêtements de la femme sont souvent axées sur leur apparence physique, y compris la nudité et les ornements, Sagard et Marie mettent en scène, sans le savoir, le regard des Autochtones sur la culture européenne. L'anecdote de la ceinture dans le texte de Sagard et du voile dans la lettre de Marie montrent bien que la curiosité et les problèmes de compréhensions mutuelles apparaissaient déjà à cette époque. Ces exemples laissent aussi entendre que la culture européenne a bien été remise en question, et que les explorateurs et missionnaires ont dû se justifier face à l'autre, avant, bien malheureusement, de s'imposer. Les réflexions des Européens sur le rôle des femmes autochtones laissent aussi entendre que l'on était conscient de l'importance de la femme dans la vie quotidienne de sa tribu. Sagard et Léry n'hésitent pas à comparer les femmes autochtones et les femmes françaises, souvent au détriment de ces dernières, et Marie semble avoir découvert le rôle traditionnel de la femme autochtone dans la religion. Comme narratrice, elle devient la voix des jeunes filles autochtones en représentant leur réalité et leur histoire personnelle. Elle leur donne alors un rôle important dans la société française où elles deviennent un modèle héroïque métissé. Même s'il s'agit pour Marie d'illustrer la foi des Autochtones, elle intègre les histoires de ces femmes à la grande Histoire. Les représentations des femmes autochtones de la Renaissance que nous avons analysées vont parfois se transformer en clichés ou en stéréotypes avant de se rendre jusqu'à nous. En revanche, nous pouvons croire que les premiers contacts entre Français et Européens laissent entendre qu'il y aurait peut-être eu un certain dialogue entre les cultures dans les premiers temps de la rencontre. Malheureusement, ce temps où le regard n'était pas totalement obscurci par le chauvinisme de la conquête et de la colonisation n'a pas duré. Peut-être pourraiton en revanche essayer d'imaginer comment ces premières observations et ces quelques éléments de véritables dialogues culturels pourraient aujourd'hui contribuer à la réconciliation.

\section{Ouvrages cités}

Compagnon, Antoine. La littérature, pour quoi faire ? Paris : Collège de France, 2007.

Deslandres, Dominique. «Altérité, identité et rédemption». Femme, mystique et missionnaire Marie Guyart de L'Incarnation. Dir. Raymond Brodeur. Québec : PU Laval, 2001. 55-68. 
Gourdeau, Claire. «Le rôle social du pensionnat des ursulines de Québec au temps de Marie de l'Incarnation ». Femme, mystique et missionnaire Marie Guyart de L'Incarnation. Dir. Raymond Brodeur. Québec : PU Laval, 2001. 145-154.

King, Thomas. The Inconvenient Indian : A Curious Account of Native People in North America. Toronto : Anchor, 2012.

Kulagina, Olga. «Représentations de l'étranger dans les récits de voyageurs français du XVII siècle ». Convergences francophones 4 (2007) : 61-73.

Lacroix, Laurier, « L'art des Huronnes vu par le frère récollet Gabriel Sagard en 1623-1624 ». Les cahiers des dix 66 (2012) : 323-338.

Lazard, Madeleine. Les avenues de Fémynie : les femmes et la Renaissance. Paris : Fayard, 2001. Léry, Jean de. Histoire d'un voyage faict en la terre du Brésil. Paris : Lestringant, 1994.

Lescarbot, Marc. Histoire de la Nouvelle France. Dir. Adrian Perier. Paris, 1617. http://gallica.bnf.fr/ark:/12148/bpt6k1095053/f2.image. 5 décembre 2017.

Marie de L'Incarnation. Correspondances. Solesmes : Abbaye Saint-Pierre de Solesmes, 1971.

Sagard, Gabriel. Le grand voyage au pays des Hurons. Québec : Bibliothèque Québécoise, 2007.

\footnotetext{
Notes

${ }^{1}$ Sagard fait partie de l'Ordre des Récollets, des missionnaires qui ont comme objectif de propager la religion catholique aux non-croyants. Les Récollets se sont installés en Nouvelle-France en 1615 et Sagard va rejoindre ses frères en 1623 (Sagard 13).

${ }^{2}$ Pendant la Renaissance, le mot « sauvage » est utilisé pour définir l'habitant des forêts, mais aujourd'hui ce mot est un terme péjoratif. En lisant les textes aujourd'hui, nous sommes décontenancés par ce mot qui est utilisé pour décrire un peuple. En effet, ce terme offensant ne signifie plus « l'habitant des forêts », mais est employé avec une connotation négative. Historiquement, il dépeint des personnes non civilisées, barbares, féroces, brutales et souvent un peuple conquit par les colonisateurs.

${ }^{3}$ «Ursuline » est un terme qui désigne une sœur religieuse. Gourdeau explique que « les congrégations d'ursulines sont un ordre enseignant essentiellement urbaine » (145) comme moyen de convertir les Autochtones au catholicisme. ${ }^{4}$ Je traduis : L'Amérique du Nord a toujours été associée au peuple autochtone, mais malgré l'histoire commune aux deux, l'Amérique du Nord ne perçoit plus les Autochtones pour ce qu'ils sont. On ne voit que des coiffes de guerre, des chemises perlées, des robes à franges en peau de cerf, des pagnes, des bandeaux, des lances à plumes, des tomahawks, des mocassins, des visages peints et des colliers en os.

${ }^{5}$ Le terme « pétun » représente le terme « tabac » aujourd'hui.
} 
